\title{
Long non-coding RNA cyclin-dependent kinase inhibitor 2B antisense ribonucleic acid 1 is associated with in-stent restenosis and promotes human carotid artery smooth muscle cell proliferation and migration by sponging miR-143-3p
}

\author{
HUANHUAN MA and AIQIN DONG
}

\author{
Department of Neurology, Cangzhou Central Hospital, Cangzhou, Hebei 061000, P.R. China
}

Received August 21, 2020; Accepted December 22, 2020

DOI: $10.3892 /$ etm.2021.9665

\begin{abstract}
Carotid angioplasty and stenting (CAS) is an efficient therapeutic approach for carotid stenosis. However, in-stent restenosis (ISR) frequently occurs and seriously affects the therapeutic efficacy of CAS. Certain non-coding (nc)RNAs serve potential roles in ISR development and progression. Thus, the goals of the present study were to investigate novel biomarkers for ISR development and to further uncover the mechanisms underlying the progression of ISR. The expression of long ncRNA cyclin-dependent kinase inhibitor (CDKN)2B-antisense 1 (AS1) and microRNA (miR)-143-3p in patients with ISR and human carotid artery smooth muscle cells (hHCtASMCs) was analyzed using reverse transcription-quantitative PCR. A luciferase reporter assay was performed to examine the interaction between CDKN2B-AS1 and miR-143-3p. The effects of the CDKN2B/miR-143-3p axis on hHCtASMC proliferation and migration were assessed using Cell Counting Kit-8 and Transwell assays. The results indicated that serum CDKN2B-AS1 was increased and miR-143-3p was decreased in patients with ISR as compared with that in patients with no ISR (all $\mathrm{P}<0.001)$. CDKN2B-AS1 and miR-143-3p were identified as risk factors for ISR onset (all P<0.05) and knockdown of CDKN2B-AS1 in hHCtASMCs led to inhibited cell proliferation and migration. Furthermore, the luciferase reporter assay and expression analysis indicated that miR-143-3p is a target of CDKN2B-AS1 and may mediate the effects of CDKN2B-AS1 on hHCtASMC proliferation and migration. In conclusion, dysregulation of CDKN2B-AS1 and miR-143-3p may represent risk factors for the occurrence of ISR. The in vitro results suggested that the CDKN2B-AS1/miR-143-3p axis may regulate the proliferation
\end{abstract}

Correspondence to: Dr Aiqin Dong, Department of Neurology, Cangzhou Central Hospital, 16 Xinhua West Road, Cangzhou, Hebei 061000, P.R. China

E-mail: aiqin_drdong@163.com

Key words: carotid stenosis, CDKN2B-AS1, miR-143-3p, in-stent restenosis, vascular smooth muscle cells, proliferation, migration and migration of hHCtASMCs, indicating its potential to be developed as a target for preventative measures and therapies for ISR.

\section{Introduction}

Carotid stenosis is caused by atherosclerosis, characterized by the accumulation of plaques accumulating in the artery wall, thus occluding the flow of blood (1). In Western countries, carotid artery stenosis accounts for approximately $20-30 \%$ of cerebral infarction cases (2). It is one of the main risk factors for the appearance of cerebral infarction (3). More than $60 \%$ of cerebral infarctions are caused by carotid stenosis and severe cerebral infarction may lead to disability or even death (4). It is also a major risk factor for stroke that leads to brain damage (5). Therefore, carotid stenosis poses a serious health threat. At present, carotid angioplasty and stenting (CAS) is used as an efficient treatment for patients with symptomatic moderateand high-grade stenosis (6). However, the frequent occurrence of restenosis after surgery seriously affects the treatment of CAS. Thus, restenosis remains an unsolved concern following CAS treatment (7). In-stent restenosis (ISR) is the gradual renarrowing of a stented coronary artery lesion due to arterial damage with subsequent neointimal tissue proliferation (8). Increasing evidence has revealed that intimal hyperplastic lesions are correlated with ISR and involve the participation of various cells, including endothelial cells and vascular smooth muscle cells (VSMCs) $(9,10)$. VSMCs exhibit increased proliferation, thereby leading to neointimal hyperplasia and ultimately initiating the progression of various cardiovascular diseases, including ISR (11). Proliferation of VSMCs and the consequent intimal thickening caused by their migration to the intima are the major pathological mechanisms leading to ISR. Therefore, the present study aimed to explore the regulatory mechanisms of VSMC proliferation and migration and to provide novel ideas and targets for the prevention of ISR.

Previous studies have indicated that circulating microRNA (miRNA/miR)-143 levels are associated with the occurrence of ISR and may serve as novel noninvasive biomarkers for ISR $(12,13)$. Furthermore, long non-coding (lnc)RNA cyclin-dependent kinase inhibitor $2 \mathrm{~B}$ antisense RNA 1 (CDKN2B-AS1) is able to target and 
regulate miR-143-3p (14). Of note, CDKN2B-AS1 participates in the pathological process of atherosclerosis and promotes the formation of atherosclerotic plaques $(15,16)$. Atherosclerosis is one of the important causes of carotid stenosis, but whether CDKN2B-AS1 participates in restenosis after stenting by targeting miR-149-3p has remained elusive. In previous studies, CDKN2B-AS1 was indicated to regulate the biological activities of numerous types of cell, including cell proliferation, migration, invasion and apoptosis $(17,18)$. Given the importance of the biological function of VSMCs in the process of restenosis, the present study aimed to further analyze whether CDKN2B-AS1 is also able to regulate the biological activity of VSMCs, thus further revealing the function of CDKN2B-AS1 and providing a novel biomarker and therapeutic target for restenosis after stenting.

\section{Materials and methods}

Patient inclusion and sample collection. A total of 52 patients who underwent CAS for carotid stenosis at Cangzhou Central Hospital (Cangzhou, China) between February 2014 and October 2016 were analyzed, including 20 patients with ISR (ISR group) and 32 patients without restenosis (non-ISR group). The degree of stenosis was determined using the criteria of the North American Symptomatic Carotid Endarterectomy trial and restenosis was based on stenosis of $\geq 50 \%$ of the stented segment (19). None of the patients had any acute infection, chronic inflammation, severe liver or kidney diseases or malignant tumors. Blood samples were collected from subjects after $\mathrm{CAS}$ and serum was centrifuged and stored at $-80^{\circ} \mathrm{C}$ for future use. The demographic and clinicopathological characteristics of the patients were recorded, including age, sex, body mass index (BMI), smoking history, drinking history, diabetes, hypertension, hyperlipidemia, total cholesterol (TC), triglyceride (TG), high-density lipoprotein cholesterol (HDL-C) and low-density lipoprotein cholesterol (LDL-C). Written informed consent was obtained from each patient and the experimental procedures were approved by the Ethics Committee of Cangzhou Central Hospital (Cangzhou, China; no. $\mathrm{CZCH} 14 \mathrm{~h} 0283$ ).

Cell culture and transfection. Human carotid artery smooth muscle cells (cat. no. 3514-05a; hHCtASMCs; Cell Applications, Inc.) were cultured with Medium 231 with Smooth Muscle Growth Supplement (Thermo Fisher Scientific, Inc.) and placed in an incubator containing $5 \% \mathrm{CO}_{2}$ at $37^{\circ} \mathrm{C}$.

Cell transfection was applied in the present study to achieve in vitro regulation of CDKN2B-AS1 and miR-143-3p. Small interfering (siRNA) for CDKN2B-AS1 (si-CDKN2B-AS1), si negative control (si-NC), mimics-NC, miR-143-3p mimics, inhibitor NC and miR143-3p inhibitor were synthesized by GenePharma and Transfection was performed when hHCtASMCs cells were grown to $80 \%$ confluence using Lipofectamine 2000 (Invitrogen; Thermo Fisher Scientific, Inc.) according to the manufacturer's protocol. Cells transfected with only transfection reagent were set as a control group. After $48 \mathrm{~h}$ of transfection, the cells were used for further analyses. The transfection sequence was as follows (from 5' to $3^{\prime}$ ): miR-143-3p mimics, UGAGAUGAAGCACUGUAGCUC; miR-143-3p inhibitor, GAGCUACAGUGCUUCAUCUCA; mimics NC, UUCUCCGAACGUGUCACGU; inhibitor NC, CAGUACUUUUGUGUAGUACAA; si-CDKN2B-AS1, UCU GUUUAAAUUAUGAAUGUG; si-NC, UCUUCCGAACGU GUCACGUTT.

RNA extraction and reverse transcription-quantitative $(R T-q) P C R$. Total RNA was extracted from fresh serum samples and cells using TRIzol reagent (Invitrogen; Thermo Fisher Scientific, Inc.). Subsequently, the RNA was reversely transcribed into single-stranded complementary DNA with the PrimeScript reverse transcriptase reagent kit (Takara Biotechnology, Inc.) following the manufacturer's protocol. The expression levels of CDKN2B-AS1 and miR-143-3p were examined by qPCR, which was performed with a SYBRGreen I Master Mix kit (Invitrogen; Thermo Fisher Scientific, Inc.) in a 7500 Real-Time PCR System (Applied Biosystems; Thermo Fisher Scientific, Inc.). U6 was used as an endogenous control for miR-143-3p and GAPDH was used as an endogenous control for CDKN2B-AS1. The following thermal cycling conditions were used for qPCR: Initial denaturation at $95^{\circ} \mathrm{C}$ for $10 \mathrm{~min}$, followed by 40 cycles of $95^{\circ} \mathrm{C}$ for $20 \mathrm{sec}, 60^{\circ} \mathrm{C}$ for $15 \mathrm{sec}$ and $72^{\circ} \mathrm{C}$ for $20 \mathrm{sec}$. The primers for qPCR were as follows: U6 forward, 5'-CTCGCTTCGGCAGCACA-3' and reverse, 5'-AACGCTTCACGAATTTGCGT-3'; miR-143-3p forward, 5'-GCCGAGTGAGATGAAGCACT-3' and reverse, 5'-CTCAACTGGTGTCGTGGA-3'; GAPDH forward, 5'-TGC ACCACCAACTGCTTAGC-3' and reverse, 5'-GGCATGCAC TGTGGTCATGAG-3'; CDKN2B-AS1 forward, 5'-ACAGAA GCCTACGAAGAACTC-3' and reverse, 5'-TGCATGGTG GTGCATCTGTA-3'. The final expression value was calculated using the $2^{-\Delta \triangle C q}$ method (20).

Cell proliferation analysis. The Cell Counting Kit-8 (CCK-8) assay was used to analyze the cell proliferation rate. Cells were seeded into a 96 -well plate $\left(5 \times 10^{3}\right.$ cells/well) and incubated for $0,24,48$ or $72 \mathrm{~h}$. Subsequently, $10 \mu \mathrm{l}$ of CCK-8 reagent (Beyotime Institute of Biotechnology, Inc.) was added to each well, followed by further incubation for $2 \mathrm{~h}$. Cell proliferation was assessed by examining the absorbance at $450 \mathrm{~nm}$ using a microplate reader.

Migration assay. Transwell chambers with $8.0 \mu \mathrm{m}$ pore size filter membranes (Corning, Inc.), without Matrigel coating (for migration assay), were used to analyze the migration abilities of hHCtASMCs. Cells in serum-free culture medium were seeded into the upper chambers and the bottom chambers were filled with medium containing $2 \%$ FBS (Gibco; Thermo Fisher Scientific, Inc.). After $48 \mathrm{~h}$ of incubation at $37^{\circ} \mathrm{C}$, cells in the lower chamber were fixed with $4 \%$ paraformaldehyde for $10 \mathrm{~min}$ at room temperature and stained with $0.1 \%$ crystal violet for $20 \mathrm{~min}$ at room temperature. The number of migrated or invaded cells in five randomly selected fields was counted under an inverted light microscope (Olympus Corp.).

Luciferase reporter assay. It was found that miR-143-3p contains a binding site for CDKN2B-AS1 through the use of LncBase Predicted v.2 (http://carolina.imis.athena-innovation. gr/diana_tools/web/index.php? $r=\operatorname{lncbasev} 2 \% 2$ Findex) and a luciferase reporter assay was used to confirm the interaction between miR-143-3p and CDKN2B-AS1 (14). The wild-type 

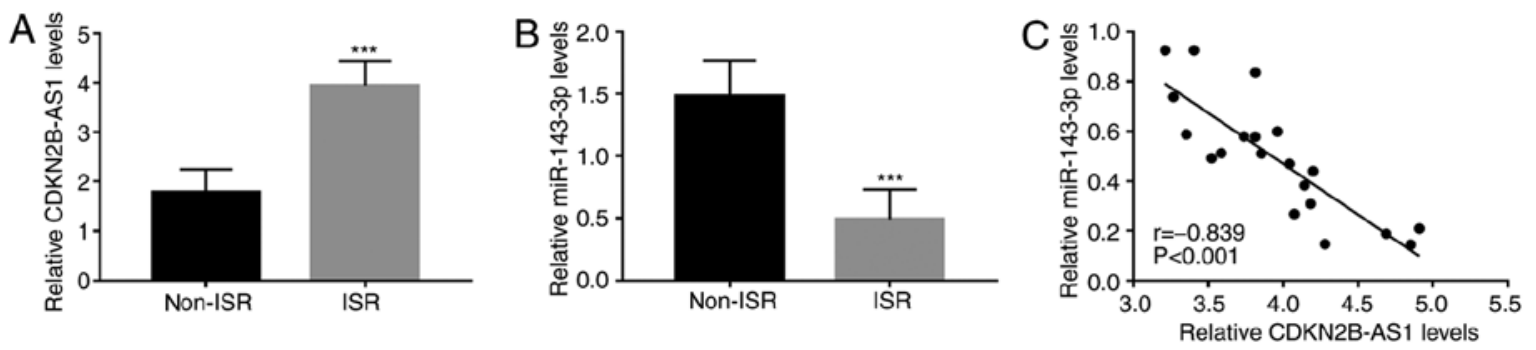

Figure 1. Expression of CDKN2B-AS1 and miR-143-3p in patients with ISR. (A) CDKN2B-AS1 levels were higher in patients with ISR than those in patients without ISR. (B) The expression of miR-143-3p was downregulated in the serum of patients with ISR compared with that in the serum of patients without ISR. (C) Serum CDKN2B-AS1 and miR-143-3p levels were significantly negatively correlated in patients with ISR. ${ }^{* * *} \mathrm{P}<0.001$ vs. non-ISR. CDKN2B-AS1, cyclin-dependent kinase inhibitor 2B-antisense 1; miR, microRNA; ISR, in-stent restenosis.

(WT) sequence of CDKN2B-AS1 contained the binding site of miR-143-3p was amplified by PCR, and the mutant-type (MUT) sequence was obtained using a QuickMutation kit (Beyotime Institute of Biotechnology). The obtained sequences were combined into a luciferase reporter vector (pGL3-luciferase basic vector; Promega Corp.). The vectors constructed were then co-transfected into hHCtASMC with miR-143-3p mimics or mimics NC using Lipofectamine 3000 (Invitrogen; Thermo Fisher Scientific, Inc.), according to the manufacturer's protocol. Changes in luciferase activity, as expected in the WT group with overexpression of miR-143-3p, were analyzed by a Dual-Luciferase Reporter Assay System (Promega Corp.) and normalized to Renilla luciferase activity.

Statistical analysis. Values are expressed as the mean \pm standard deviation and analyzed using SPSS 21.0 (IBM Corp.) and GraphPad 7.0 (GraphPad Software, Inc.). Differences between groups were analyzed with Student's t-test, the chi-square test or analysis of variance with Tukey's post-hoc test. Pearson correlation analysis was used to analyze the correlation between the expression of CDKN2B-AS1 and miR-143-3p. Logistic regression was used to analyze the association of factors such as CDKN2B-AS1 and miR-143-3p with the occurrence of ISR. $\mathrm{P}<0.05$ was considered to indicate statistical significance.

\section{Results}

Baseline characteristics of the study subjects. The Student's t-test and chi-square test were used to compare the basic data of the two groups of patients. Among the patients, there were 26 males and 6 females with a mean age of $64.0 \pm 7.3$ years in the non ISR group; and 17 males and 3 females with a mean age of $67.5 \pm 8.5$ years in the ISR group. The results indicated that there were no differences in age, sex, BMI, smoking, drinking, hypertension, hyperlipidemia, TG or HDL-C between the two groups (all $\mathrm{P}>0.05$ ). However, more cases of diabetes, higher TC levels and lower LDL-C levels were present in the ISR group as compared with those in the non-ISR group (all $\mathrm{P}<0.05$; Table I).

Expression of CDKN2B-AS1 and miR-143-3p in patients with ISR. To further elucidate the roles of CDKN2B-AS1 and miR-143-3p in ISR, the expression of CDKN2B-AS1 and miR-143-3p in the serum of patients with ISR was quantified
Table I. Baseline characteristics of the study subjects.

\begin{tabular}{lccc}
\hline Variables & non-ISR $(\mathrm{n}=32)$ & ISR $(\mathrm{n}=20)$ & P-value \\
\hline Age (years) & $64.0 \pm 7.3$ & $67.5 \pm 8.5$ & 0.129 \\
Male sex & 26 & 17 & 0.728 \\
BMI $\left(\mathrm{kg} / \mathrm{m}^{2}\right)$ & $24.5 \pm 2.6$ & $25.1 \pm 3.1$ & 0.449 \\
Smoking history & 13 & 9 & 0.756 \\
Drinking history & 13 & 10 & 0.508 \\
Diabetes & 10 & 10 & 0.041 \\
Hypertension & 21 & 16 & 0.266 \\
Hyperlipidemia & 13 & 11 & 0.312 \\
TC $(\mathrm{mg} / \mathrm{dl})$ & $147.6 \pm 35.7$ & $169.88 \pm 36.9$ & 0.036 \\
TG $(\mathrm{mg} / \mathrm{dl})$ & $140.3 \pm 48.4$ & $134.6 \pm 48.0$ & 0.680 \\
HDL-C $(\mathrm{mg} / \mathrm{dl})$ & $39.9 \pm 6.5$ & $41.7 \pm 7.1$ & 0.367 \\
LDL-C $(\mathrm{mg} / \mathrm{dl})$ & $96.6 \pm 19.7$ & $84.2 \pm 18.5$ & 0.028
\end{tabular}

Values are expressed as the mean \pm standard deviation or $\mathrm{n}$. BMI, body mass index; TC, total cholesterol; TG, triglyceride; HDL-C, high-density lipoprotein cholesterol; LDL-C, low-density lipoprotein cholesterol; ISR, in-stent restenosis.

by RT-qPCR. The results indicated that compared with those in the non-ISR group, serum CDKN2B-AS1 was upregulated in the ISR group ( $\mathrm{P}<0.001 ;$ Fig. $1 \mathrm{~A})$, while serum miR-143-3p was downregulated ( $\mathrm{P}<0.001$; Fig. 1B). Furthermore, the serum levels of CDKN2B-AS1 and miR-143-3p were significantly negatively correlated in patients with ISR ( $\mathrm{r}=-0.839, \mathrm{P}<0.001$; Fig. 1C).

Association of CDKN2B-AS1 and miR-143-3p with the occurrence of ISR. The clinical data of all patients and the expression levels of CDKN2B-AS1 and miR-143-3p were included in the univariate and multivariate logistic regression analysis. The univariate analysis results indicated that diabetes, hypertension, TC, LDL-C, miR-143-3p and CDKN2B-AS1 (all $\mathrm{P}<0.05)$ were related with ISR onset and the further multivariate analysis data demonstrated that CDKN2B-AS1 $(\mathrm{P}=0.003)$, miR-143-3p $(\mathrm{P}<0.001)$ and LDL-C $(\mathrm{P}=0.034)$ were independently associated with the occurrence of ISR and were potential risk factors for the occurrence of ISR (Table II).

Knockdown of CDKN2B-AS1 inhibits the proliferation and migration of hHCtASMCs. The regulatory effects of 
Table II. Logistic regression analysis results for patients with ISR.

\begin{tabular}{|c|c|c|c|c|c|c|}
\hline \multirow[b]{2}{*}{ Variables } & \multicolumn{3}{|c|}{ Univariate analysis } & \multicolumn{3}{|c|}{ Multivariate analysis } \\
\hline & OR & $95 \% \mathrm{CI}$ & P-value & OR & $95 \% \mathrm{CI}$ & $\mathrm{P}$-value \\
\hline Age $(65.3 \pm 7.9)$ & 1.428 & $0.894-2.016$ & 0.228 & 1.229 & $0.812-2.285$ & 0.268 \\
\hline Sex (male vs. female) & 1.094 & $0.639-1.598$ & 0.712 & 1.006 & $0.986-1.025$ & 0.872 \\
\hline BMI $(24.7 \pm 2.8)$ & 1.598 & $0.921-2.228$ & 0.114 & 1.612 & $0.809-2.998$ & 0.135 \\
\hline Smoking (yes vs. no) & 1.188 & $0.618-1.849$ & 0.662 & 1.126 & $0.525-3.196$ & 0.627 \\
\hline Drinking (yes vs. no) & 1.213 & $0.685-1.941$ & 0.589 & 2.193 & $0.751-4.231$ & 0.660 \\
\hline Diabetes (yes vs. no) & 1.666 & $1.045-2.285$ & 0.044 & 1.630 & $0.823-2.512$ & 0.254 \\
\hline Hypertension (yes vs. no) & 1.687 & $1.101-2.318$ & 0.038 & 2.507 & $0.912-4.285$ & 0.113 \\
\hline Hyperlipidemia (yes vs. no) & 1.108 & $0.547-1.799$ & 0.697 & 1.171 & $0.988-1.842$ & 0.895 \\
\hline TC $(156.2 \pm 37.4)$ & 1.691 & $1.112-2.484$ & 0.035 & 2.834 & $0.979-5.109$ & 0.092 \\
\hline 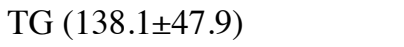 & 1.674 & $0.994-2.485$ & 0.051 & 1.948 & $0.894-3.785$ & 0.330 \\
\hline HDL-C (40.6 \pm 6.8$)$ & 1.192 & $0.640-1.886$ & 0.610 & 1.062 & $0.912-1.285$ & 0.803 \\
\hline LDL-C (91.8 \pm 20.0$)$ & 2.085 & $1.397-3.896$ & 0.011 & 2.113 & $1.428-4.219$ & 0.034 \\
\hline miR-143-3p (2.6 \pm 1.2$)$ & 2.969 & $1.941-4.573$ & $<0.001$ & 3.096 & $1.926-4.899$ & $<0.001$ \\
\hline CDKN2B-AS1 $(1.1 \pm 0.6)$ & 2.818 & $1.881-4.141$ & 0.002 & 2.854 & $1.701-4.511$ & 0.003 \\
\hline
\end{tabular}

The OR refers to the subject having ISR. BMI, body mass index; TC, total cholesterol; TG, triglyceride; HDL-C, high-density lipoprotein cholesterol; ISR, in-stent restenosis; LDL-C, low-density lipoprotein cholesterol; OR, odds ratio; miR, microRNA; CDKN2B-AS1, cyclin-dependent kinase inhibitor 2B-antisense 1.
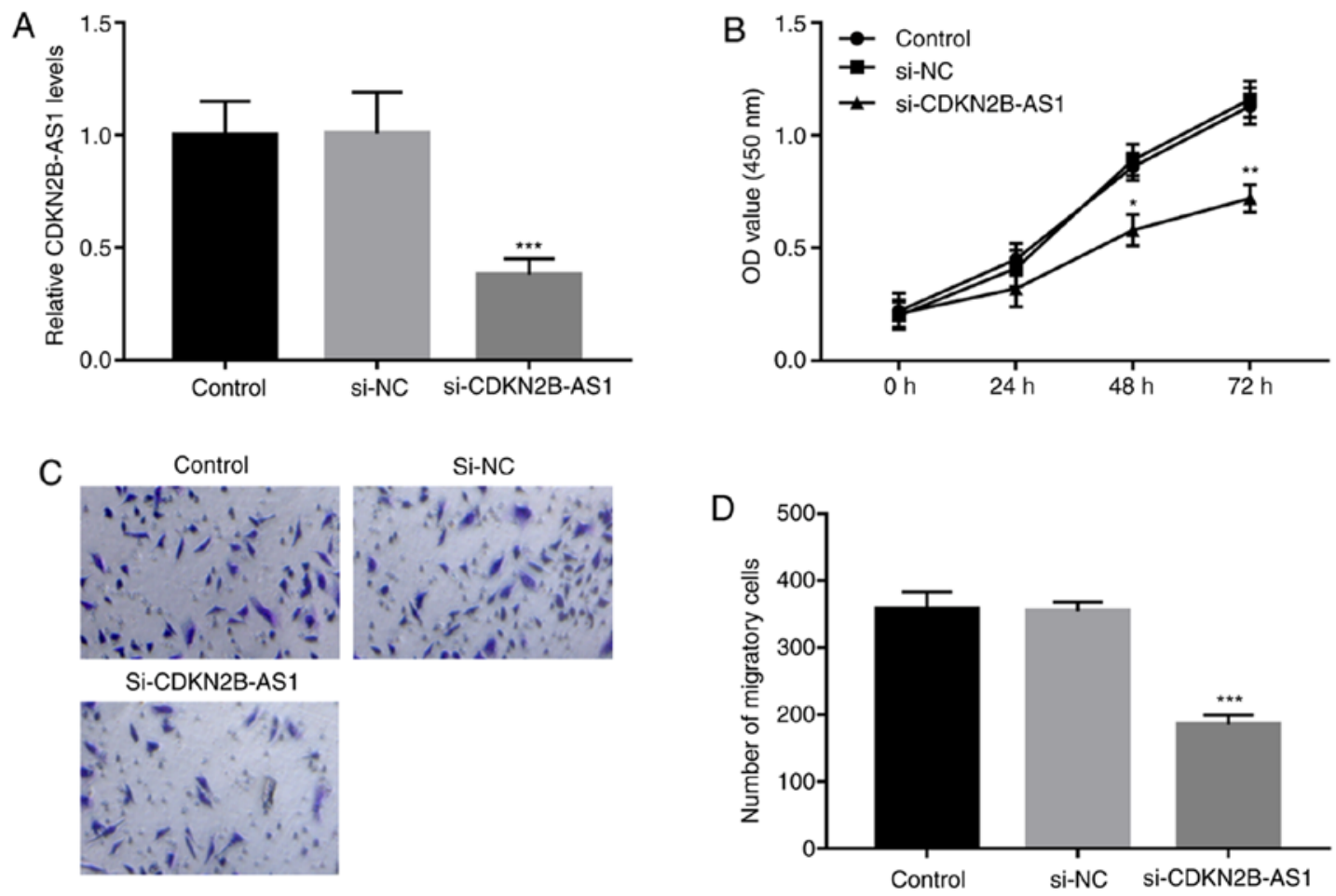

Figure 2. Knockdown of CDKN2B-AS1 inhibits cell proliferation and migration of hHCtASMCs. (A) The expression of CDKN2B-AS1 was significantly lower following transfection with si-CDKN2B-AS1. (B) The proliferation of hHCtASMCs was suppressed by si-CDKN2B-AS1. (C and D) The migratory ability of hHCtASMCs was inhibited by knockdown of CDKN2B-AS1. (C) Representative images of the lower sides of the membranes with migrated cells (magnification, $\mathrm{x} 100$; stained with $0.1 \%$ crystal violet) and (D) quantified numbers of migrated cells in each group. ${ }^{*} \mathrm{P}<0.05,{ }^{* * *} \mathrm{P}<0.01$ and ${ }^{* * *} \mathrm{P}<0.001$ vs. the control group. si-CDKN2B-AS1, short interfering RNA targeting cyclin-dependent kinase inhibitor 2B-antisense 1; hHCtASMCs, human carotid artery smooth muscle cells; OD, optical density; NC, negative control.

CDKN2B-AS1 on cell proliferation and migration as part of the pathology of ISR were analyzed in hHCtASMCs cells.
The expression levels of CDKN2B-AS1 in hHCtASMCs were confirmed to be significantly downregulated by the 
CDKN2B-AS1 WT 5'-...AAGAAAGGAAAGCGAGGUCAUCUCA-...-3'

miR-143-3p

$\|||||||$

CDKN2B-AS1 MUT 5'-...AAGAAAGGAAGGGAGGAGUAGAGU-...-3'
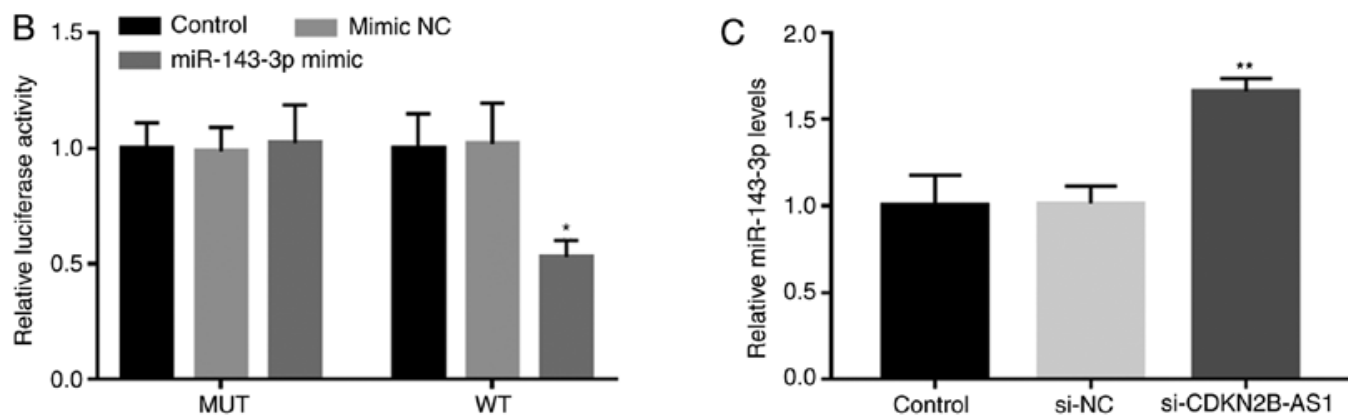

Figure 3. CDKN2B-AS1 directly inhibits miR-143-3p expression in hHCtASMCs. (A) The complementary sequence of miR-143-3p in CDKN2B-AS1. (B) In hHCtASMCs, the luciferase activity of the reporter plasmid carrying CDKN2B-AS1-WT was decreased by miR-143-3p overexpression. (C) Knockdown of CDKN2B-AS1 by si-CDKN2B-AS1 enhanced the expression of miR-143-3p levels in hHCtASMCs. ${ }^{*} \mathrm{P}<0.05,{ }^{* * *} \mathrm{P}<0.01$ vs. the control group. si-CDKN2B-AS1, short interfering RNA targeting cyclin-dependent kinase inhibitor 2B-antisense 1; hHCtASMCs, human carotid artery smooth muscle cells; WT, wild-type; MUT, mutant; miR, microRNA; NC, negative control.
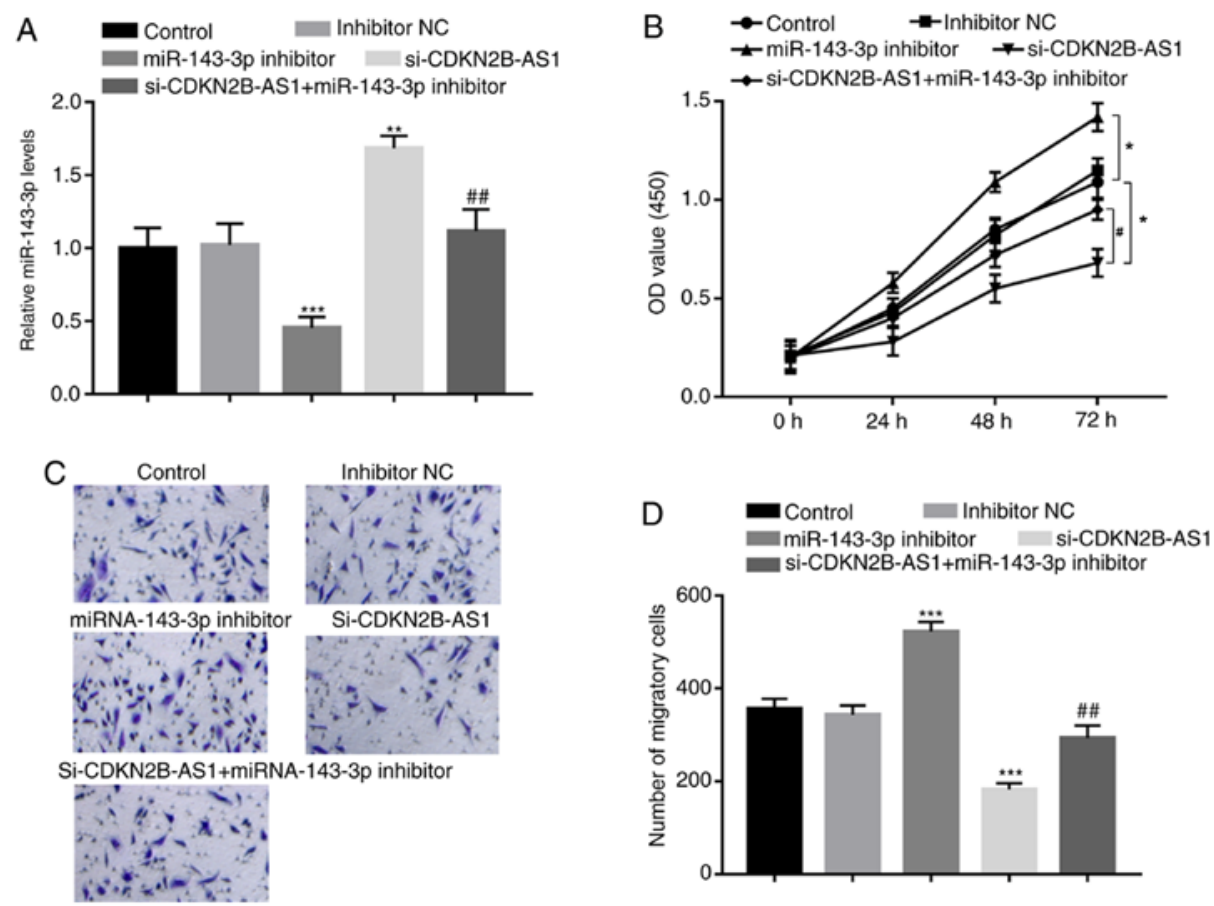

Figure 4. miR-143-3p mediates the effects of CDKN2B-AS1 on hHCtASMC proliferation and migration. (A) In hHCtASMCs, the expression of miR-143-3p was suppressed by miR-143-3p inhibitor but was promoted by si-CDKN2B-AS1 and the increase of miR-143-3p by si-CDKN2B-AS1 was also inhibited by the miR-143-3p inhibitor. (B) Effect of si-CDKN2B-AS1 and miR-143-3p inhibitors on the proliferation of hHCtASMC. (C) The representative images of migrated cells (magnification, $x 100$; stained by $0.1 \%$ crystal violet). (D) Quantified number of migrated cells in each group. ${ }^{*} \mathrm{P}<0.05,{ }^{* *} \mathrm{P}<0.01$ and ${ }^{* * *} \mathrm{P}<0.001$ vs. the control group; ${ }^{\#} \mathrm{P}<0.05$ and ${ }^{\# \#} \mathrm{P}<0.01$ vs. si-CDKN2B-AS1. si-CDKN2B-AS1, short interfering RNA targeting cyclin-dependent kinase inhibitor $2 \mathrm{~B}$-antisense 1; hHCtASMCs, human carotid artery smooth muscle cells; OD, optical density; NC, negative control; miR/miRNA, microRNA.

si-CDKN2B-AS1 (P<0.001; Fig. 2A). The results of CCK-8 and Transwell assays suggested that the proliferation and migration of the cells were significantly inhibited after silencing CDKN2B-AS1 (all P<0.05; Fig. 2B-D).

CDKN2B-AS1 directly inhibits miR-143-3p expression in hHCtASMCs. The complementary sequence of miR-143-3p in the CDKN2B-AS1 sequence is presented in
Fig. 3A. The results of the luciferase assay demonstrated that CDKN2B-AS1 and miR-143-3p were able to directly interact, which manifested as a significantly decreased luciferase activity following miR-143-3p overexpression in the WT CDKN2B-AS1 group ( $\mathrm{P}<0.05$; Fig. 3B). Furthermore, the RT-qPCR results indicated that the silencing of CDKN2B-AS1 in hHCtASMCs significantly promoted the expression of miR-143-3p, indicating that CDKN2B-AS1 is 
able to directly interfere with the expression of miR-143-3p in hHCtASMCs $(\mathrm{P}<0.01$; Fig. $3 \mathrm{C})$.

miR-143-3p mediates the effects of CDKN2B-AS1 on $h H C t A S M C$ proliferation and migration. To further confirm the regulatory effect of miR-143-3p on the biological function of CDKN2B-AS1, the expression levels of CDKN2B-AS1 and miR-143-3p were co-regulated in hHCtASMCs. miR-143-3p inhibitor was confirmed to significantly inhibit the expression of miR-143-3p in hHCtASMCs ( $\mathrm{P}<0.001$; Fig. 4A), which led to marked increases in hHCtASMC proliferation and migration (all P<0.05; Fig. 4B-D). Furthermore, in cells co-transfected with si-CDKN2B-AS1 and miR-143-3p inhibitor, the increase in miR-143-3p expression induced by CDKN2B-AS1 knockdown alone was significantly decreased ( $\mathrm{P}<0.01$; Fig. 4A). In addition, CDKN2B-AS1 knockdown-induced inhibition of hHCtASMC proliferation and migration were abrogated by simultaneous downregulation of miR-143-3p (all $\mathrm{P}<0.05$; Fig. 4B-D), indicating that miR-143-3p may mediate the regulatory effects of CDKN2B-AS1 on hHCtASMC proliferation and migration.

\section{Discussion}

Carotid stenosis refers to the narrowing or contraction of the internal surface of the carotid artery (21). It may result in brain tissue injury, which is associated with intracranial arterial flow disturbances, as well as microembolic complications (22). Carotid stenosis seriously threatens human health. CAS has become one of the important means of treating carotid stenosis (23), but there is a certain proportion of ISR after stent implantation that causes problems in the clinic (24). ISR seriously affects the efficacy of CAS. Of note, abnormal expression of certain miRNAs and lncRNAs in ISR has been discovered. For instance, miR-93-5p was reported to have cardiac biomarker potential as a predictor of ISR (25). Furthermore, Jiang et al (24) indicated that miR-17 is abnormally expressed in patients with ISR and has potential as a biomarker for ISR. The expression of lncRNA antisense non-coding RNA in the INK4 locus (ANRIL) was reported to be associated with ISR, indicating that ANRIL may be a suitable prognostic factor for ISR (26). IncRNA CDKN2B-AS1 was previously reported to affect cancer progression by regulating miRNAs. For instance, overexpression of CDKN2B-AS1 resulted in the promotion of cell proliferation, invasion, migration and inhibition of apoptosis and senescence of cervical cancer cells by regulating the miR181a-5p/TGF 31 axis (27). Furthermore, CDKN2B-AS1 promoted hepatocellular carcinoma growth and metastasis by promoting nucleosome assembly protein 1 like 1-mediated PI3K/AKT/mTOR signaling via acting as a molecular sponge of let-7c-5p (17). However, the biological functions of CDKN2B-AS1 and miRNA-143-3p in ISR remain unclear. In the present study, it was determined that CDKN2B-AS1 and miR-143-3p were abnormally expressed in ISR. The results suggested that compared to patients in the non-ISR group, patients in the ISR group had upregulated serum levels of CDKN2B-AS1 and downregulated serum levels of miR143-3p. Furthermore, the expression levels of CDKN2B-AS1 and miR-143-3p in serum were significantly negatively correlated in patients with ISR. The results of the logistic multivariate regression analysis indicated that CDKN2B-AS1 and miR-143-3p were independently associated with the occurrence of ISR and are potential risk factors for the occurrence of ISR.

VSMCs are the major cell type in blood vessels (28). Abnormal proliferation and migration of VSMCs are related to ISR (29). Certain studies have indicated that IncRNA may affect the proliferation and migration of VSMCs. For instance, lncRNA taurine-upregulated gene 1/miR-145-5p/fibroblast growth factor 10 was reported to promote the proliferation and migration of VSMCs in the hypertensive state by activating the Wnt/ $\beta$-catenin pathway (30). Tang et al (31) demonstrated for the first time that IncRNA growth arrest specific 5 negatively impacts VSMC survival via activation of the p53 pathway during vascular remodeling. However, the relationship between CDKN2B-AS1 and miR-143-3p and VSMCs had remained elusive. In the present study, RT-qPCR was used to measure the expression levels of CDKN2B-AS1 in hHCtASMCs and it was confirmed that CDKN2B-AS1 expression was downregulated by si-CDKN2B-AS1. It was determined that silencing of CDKN2B-AS1 inhibited the migration and proliferation of hHCtASMCs cells. Therefore, it was speculated that CDKN2B-AS1 has a promoting role in the development of ISR. It was identified that miR-143-3p is able to bind with CDKN2B-AS1 (14). A luciferase reporter assay confirmed that CDKN2B-AS1 is able to specifically associate with miR-143-3p in hHCtASMCs. Furthermore, it was demonstrated that inhibition of miR-143-3p was able to abrogate the effect of CDKN2BAS1 knockdown to inhibit the proliferation and migration of hHCtASMCs. Thus, it was suggested that CDKN2B-AS1 may directly regulate miR-143-3p in hHCtASMCs.

In conclusion, in the present study, CDKN2B-AS1 expression levels were determined to be upregulated while miR-143-3p expression levels were downregulated in hHCtASMCs, and CDKN2B-AS1 and miR-143-3p were independently associated with the occurrence of ISR, which were thus suggested to be potential risk factors for the occurrence of ISR. In addition, silencing of CDKN2B-AS1 inhibited the proliferation and migration of hHCtASMC by targeting miR-143-3p. Thus, aberrant CDKN2B-AS1 and miR-143-3p expression may be involved in the development and progression of ISR via regulating biological functions of VSMCs. Overall, the present study may provide novel non-invasive biomarkers for ISR prevention and treatment and novel insight into the pathogenesis of ISR development. However, there are still certain limitations to the present study. For instance, changes of the CDKN2B-AS1/miR-143-3p axis in the lesion tissue samples were not assessed. Therefore, further studies are required in the future to confirm the role of CDKN2B-AS1/miR-143-3p in ISR lesion tissue, and the exact mechanisms remain to be explored.

\section{Acknowledgements}

Not applicable.

\section{Funding}

No funding was received. 


\section{Availability of data and materials}

The datasets used and/or analyzed during the current study are available from the corresponding author on reasonable request.

\section{Authors' contributions}

HM analyzed and interpreted the data. HM and AD performed the cell experiments and blood analysis, wrote and revised the manuscript, and checked and confirm the authenticity of the raw data. Both authors read and approved the final manuscript.

\section{Ethics approval and consent to participate}

Written informed consent was obtained from each patient and the experimental procedures were approved by the Ethics Committee of Cangzhou Central Hospital (Cangzhou, China; approval no. CZCH14h0283).

\section{Patient consent for publication}

Not applicable.

\section{Competing interests}

The authors declare that they have no competing interests.

\section{References}

1. Meschia JF, Klaas JP, Brown RD Jr and Brott TG: Evaluation and management of atherosclerotic carotid stenosis. Mayo Clin Proc 92: 1144-1157, 2017.

2. Sarikas A, Carrier L, Schenke C, Doll D, Flavigny J, Lindenberg KS, Eschenhagen T and Zolk O: Impairment of the ubiquitin-proteasome system by truncated cardiac myosin binding protein C mutants. Cardiovasc Res 66: 33-44, 2005.

3. Cheng SF, Brown MM, Simister RJ and Richards T: Contemporary prevalence of carotid stenosis in patients presenting with ischaemic stroke. Br J Surg 106: 872-878, 2019.

4. De Reuck JL: Pathophysiology of carotid artery disease and related clinical syndromes. Acta Chir Belg 104: 30-34, 2004.

5. Sherman DG: The carotid artery and stroke. Am Fam Phys 40 (5 Suppl): 41S-44S, 7S-9S, 1989.

6. Fanelli F, Boatta E, Cannavale A, Corona M, Lucatelli P, Wlderk A, Cirelli $\mathrm{C}$ and Salvatori FM: Carotid artery stenting: Analysis of a 12-year single-center experience. J Endovasc Ther 19: 749-756, 2012.

7. AbuRahma AF, Abu-Halimah S, Hass SM, Nanjundappa A, Stone PA, Mousa A, Lough E and Dean LS: Carotid artery stenting outcomes are equivalent to carotid endarterectomy outcomes for patients with post-carotid endarterectomy stenosis. J Vasc Surg 52: 1180-1187, 2010.

8. Dangas GD, Claessen BE, Caixeta A, Sanidas EA, Mintz GS and Mehran R: In-stent restenosis in the drug-eluting stent era. J Am Coll Cardiol 56: 1897-1907, 2010.

9. Zhang H, Ren KF, Chang H, Wang JL and Ji J: Surface-mediated transfection of a pDNA vector encoding short hairpin RNA to downregulate TGF- $\beta 1$ expression for the prevention of in-stent restenosis. Biomaterials 116: 95-105, 2017.

10. Bagyura Z, Kiss L, Berta B, Szilágyi Á, Hirschberg K, Széplaki G, Lux Á, Szelid Z, Soós P and Merkely B: High rate of in-stent restenosis after coronary intervention in carriers of the mutant mannose-binding lectin allele. BMC Cardiovasc Disord 17: 4, 2017.

11. Santulli G: microRNAs distinctively regulate vascular smooth muscle and endothelial cells: Functional implications in angiogenesis, atherosclerosis, and in-stent restenosis. Adv Exp Med Biol 887: 53-77, 2015.

12. Yuan Y, Liu X, Hao S, He Q and Shen Z: Plasma levels of miR-143 and miR-145 are associated with coronary in-stent restenosis within 1 year of follow-up after drug-eluting stent implantation. Ann Transl Med 8: 756, 2020.
13. He M, Gong Y, Shi J, Pan Z, Zou H, Sun D, Tu X, Tan X, $\mathrm{Li} \mathrm{J}, \mathrm{Li} \mathrm{W}$, et al: Plasma microRNAs as potential noninvasive biomarkers for in-stent restenosis. PLoS One 9: e112043, 2014.

14. Xu C, Zhai J and Fu Y: LncRNA CDKN2B-AS1 promotes the progression of ovarian cancer by miR-143-3p/SMAD3 axis and predicts a poor prognosis. Neoplasma 67: 782-793, 2020.

15. Li H, Han S, Sun Q, Yao Y, Li S, Yuan C, Zhang B, Jing B, Wu J, Song $\mathrm{Y}$ and Wang H: Long non-coding RNA CDKN2B-AS1 reduces inflammatory response and promotes cholesterol efflux in atherosclerosis by inhibiting ADAM10 expression. Aging (Albany NY) 11: 1695-1715, 2019.

16. Ou M, Li X, Zhao S, Cui S and Tu J: Long non-coding RNA CDKN2B-AS1 contributes to atherosclerotic plaque formation by forming RNA-DNA triplex in the CDKN2B promoter. EBioMedicine 55: 102694, 2020.

17. Huang Y, Xiang B, Liu Y, Wang Y and Kan H: LncRNA CDKN2B-AS1 promotes tumor growth and metastasis of human hepatocellular carcinoma by targeting let-7c-5p/NAP1L1 axis. Cancer Lett 437: 56-66, 2018.

18. Zhuang H, Cao G, Kou C and Li D: Overexpressed lncRNA CDKN2B-AS1 is an independent prognostic factor for liver cancer and promotes its proliferation. J BUON 24: 1441-1448, 2019.

19. Gasecki AP, Hachinski VC, Mendel T and Barnett HT: Endarterectomy for symptomatic carotid stenosis. Review of the European and North American symptomatic carotid surgery trials. Nebr Med J 77: 121-123, 1992.

20. Livak KJ and Schmittgen TD: Analysis of relative gene expression data using real-time quantitative PCR and the 2(-Delta Delta C(T)) method. Methods 25: 402-408, 2001.

21. Chen KP, Wang JJ, Wang LJ, Lu J, Qi P, Hu S, Yang XM, Wang HF and Wang DM: Study of correlation between carotid artery tortuosity and atherosclerotic carotid artery stenosis. Zhonghua Wai Ke Za Zhi 55: 608-612, 2017 (In Chinese).

22. Puz P, Lasek-Bal A, Urbanek T and Kazibutowska Z: Assessment of cerebral embolism and vascular reserve parameters in patients with carotid artery stenosis. Neurol Neurochir 50: 356-362, 2016.

23. Wang T, Mei B and Zhang J: Atherosclerotic carotid stenosis and cognitive function. Clin Neurol Neurosurg 146: 64-70, 2016.

24. Jiang F, Zhang X, Lu YM, Li YG, Zhou X and Wang YS: Elevated level of miR-17 along with decreased levels of TIMP-1 and IL-6 in plasma associated with the risk of in-stent restenosis. Biosci Trends 13: 423-429, 2019.

25. O'Sullivan JF, Neylon A, Fahy EF, Yang P, McGorrian C and Blake GJ: MiR-93-5p is a novel predictor of coronary in-stent restenosis. Heart Asia 11: e011134, 2019.

26. Wang F, Su X, Liu C, Wu M and Li B: Prognostic value of plasma long noncoding RNA ANRIL for in-stent restenosis. Med Sci Monit 23: 4733-4739, 2017.

27. Zhu L, Zhang Q, Li S, Jiang S, Cui J and Dang G: Interference of the long noncoding RNA CDKN2B-AS1 upregulates miR-181a-5p/TGF $\beta$ I axis to restrain the metastasis and promote apoptosis and senescence of cervical cancer cells. Cancer Med 8: 1721-1730, 2019.

28. Frismantiene A, Philippova M, Erne P and Resink TJ: Smooth muscle cell-driven vascular diseases and molecular mechanisms of VSMC plasticity. Cell Signal 52: 48-64, 2018.

29. He Y, Zou P, Lu Y, Jia D, Li X, Yang H, Tang L, Zhu Z, Tu T, Tai S, et al: Osteoprotegerin promotes intimal hyperplasia and contributes to in-stent restenosis: Role of an $\alpha \mathrm{V} \beta 3 / \mathrm{FAK}$ dependent YAP pathway. J Mol Cell Cardiol 139: 1-13, 2020.

30. Shi L, Tian C, Sun L, Cao F and Meng Z: The lncRNA TUG1/miR-145-5p/FGF10 regulates proliferation and migration in VSMCs of hypertension. Biochem Biophys Res Commun 501: 688-695, 2018.

31. Tang R, Mei X, Wang YC, Cui XB, Zhang G, Li W and Chen SY: LncRNA GAS5 regulates vascular smooth muscle cell cycle arrest and apoptosis via 53 pathway. Biochim Biophys Acta Mol Basis Dis 1865: 2516-2525, 2019.

This work is licensed under a Creative Commons Attribution-NonCommercial-NoDerivatives 4.0 International (CC BY-NC-ND 4.0) License. 